\title{
Ernst Otto Bräunche, Stephan Sander-Faes (dir.), Städte im Krieg - Erlebnis, Inszenierung und Erinnerung des Ersten Weltkriegs
}

\section{Aurore Peyroles}

\section{CpenEdition}

\section{Journals}

Édition électronique

URL : http://journals.openedition.org/ifha/8833

DOI : $10.4000 /$ ifha. 8833

ISSN : 2198-8943

Éditeur

IFRA - Institut franco-allemand (sciences historiques et sociales)

Référence électronique

Aurore Peyroles, «Ernst Otto Bräunche, Stephan Sander-Faes (dir.), Städte im Krieg - Erlebnis, Inszenierung und Erinnerung des Ersten Weltkriegs », Revue de l'IFHA [En ligne], Date de recension, mis en ligne le 02 novembre 2017, consulté le 24 septembre 2020. URL : http://journals.openedition.org/ ifha/8833 ; DOI : https://doi.org/10.4000/ifha.8833

Ce document a été généré automatiquement le 24 septembre 2020.

(C)IFHA 


\title{
Ernst Otto Bräunche, Stephan
} Sander-Faes (dir.), Städte im Krieg Erlebnis, Inszenierung und Erinnerung des Ersten Weltkriegs

\author{
Aurore Peyroles
}

\section{RÉFÉRENCE}

Ernst Otto Bräunche, Stephan Sander-Faes (dir.), Städte im Krieg - Erlebnis, Inszenierung und Erinnerung des Ersten Weltkriegs (Stadt in der Geschichte, Band 41), Ostfildern:

Thorbecke, 2016, 232 p., $29 €$ 
Comment la guerre s'inscrit-elle dans la ville? C'est à cette question que tente de répondre l'ouvrage collectif issu d'un colloque organisé par le Südwestdeutschen Arbeitskreis für Stadtforschung en 2013 : en se penchant sur différentes villes, parmi lesquelles Karlsruhe et Mannheim occupent une place de choix, les éclairages successifs examinent la façon dont la Première Guerre mondiale, mais aussi sa mémoire, se sont emparées (ou non) de l'espace urbain. L'ensemble convainc par la diversité non des échelles mais des lieux traités: les capitales des grandes puissantes belligérantes (Vienne, Paris et Berlin) n'éclipsent pas les villes d'importance régionale; certaines villes sont très proches du front (Nancy, Eupen), d'autres très éloignées. Même s'ils

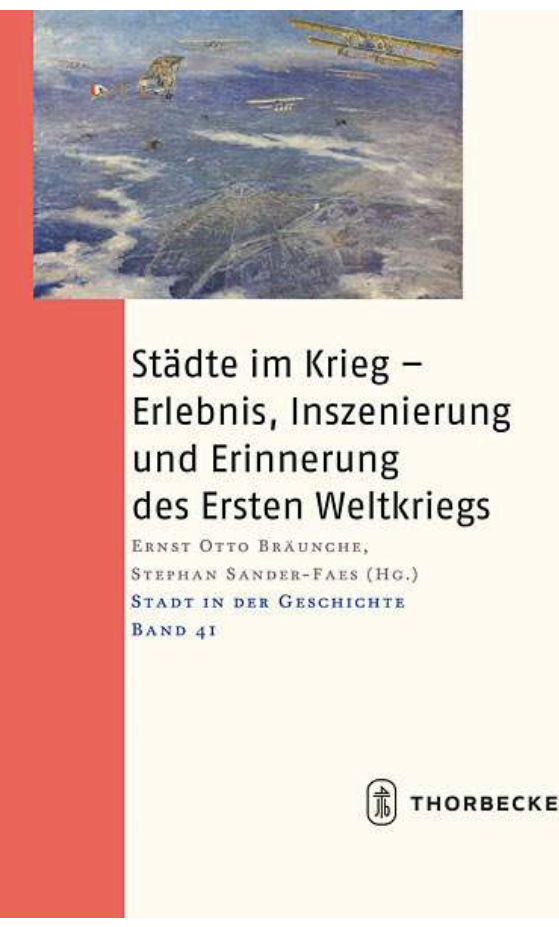
soulignent les particularités de chaque cas et mettent à jour d'importantes nuances, les articles s'attachent à montrer que la Première Guerre mondiale fut la première à pouvoir être qualifiée de "guerre totale", marquant de son empreinte tous les domaines de la vie - et de la ville.

Les premières contributions étudient le surgissement du conflit à l'été 1914 : comment réagissent les villes à l'entrée en guerre? Qu'est-ce qui se modifie immédiatement dans les physionomies, les pratiques et les habitudes urbaines? Le resserrement géographique des articles permet de rendre compte des conséquences très concrètes de la déclaration de guerre dans les villes de Karlsruhe, de Nancy, de Mannheim et d'Eupen, petite ville située à la frontière germano-belgo-néerlandaise. Que se passe-t-il dans ces villes moyennes qui se voient soudainement érigées en points stratégiques? Comment réagissent-elles au départ des hommes, mais aussi à la soudaine arrivée de dizaines de milliers de soldats, de prisonniers de guerre et de blessés?

Les récurrences sont frappantes : aux parades militaires d'août 1914 acclamées par des cris de joie et les échos du chant «Lieb Vaterland, magst ruhig sein!» succèdent les colonnes de blessés et de réfugiés. Les manifestations d'enthousiasme laissent vite place aux rumeurs les plus inquiétantes: dans les quatre villes étudiées circule en particulier le bruit que l'eau est empoisonnée ; toutes sont saisies d'une "spionitis" aigüe. Le problème le plus urgent que les autorités municipales doivent affronter est celui de l'approvisionnement, les contraignant à intervenir très directement sur la réglementation des prix, mais aussi des espaces: des terrains, y compris le stade de Karlsuhe, sont réquisitionnés pour servir de "Kriegsgärten». Surtout, les villes s'organisent pour faire face à l'afflux des blessés, réquisitionnant les principaux bâtiments pour les convertir en hôpitaux militaires. Les tableaux de ces quatre villes confirment ainsi des éléments connus, mais l'éclairage local, le plus souvent doublé de photographies, a le mérite de les rendre très concrets. Ayant recours à des extraits de 
presse, mais aussi à des documents intimes (journaux ou correspondance), la plupart des auteurs se placent au plus près de l'expérience vécue de la guerre dans ces villes.

Le point de vue local permet pourtant aussi de faire apparaître d'intéressantes nuances. Ainsi, si la vie politique de Karlsruhe, comme celle de la plupart des villes allemandes, est rapidement suspendue au nom de l'unité nationale ("Ich kenne keine Parteien, ich kenne nur Deutsche »), Franz Jungbluth estime que la "Burgfrieden» ne s'impose pas véritablement à Mannheim, l'entrée en guerre entraînant plutôt la fin d'une constellation politique municipale singulière de coopération entre socialistes et nationalistes. Se dessine surtout une expérience de la guerre différente selon la localisation géographique: Nancy est à 20 kilomètres de la frontière, le centre-ville d'Eupen à 2 kilomètres à peine. Ces villes sont directement exposées à la violence des combats, leurs habitants entendent le canon et voient le front se rapprocher. En s'appuyant sur des documents autobiographiques, Bernhard Liemann restitue ainsi la surprise des habitants d'Eupen au petit matin du 4 août quand ils voient arriver des dizaines de milliers de soldats feldgrau lancés à la conquête de la Belgique.

Un deuxième ensemble de contributions s'intéresse à la façon dont la Première Guerre mondiale s'est inscrite dans l'espace urbain entre 1914 et 1918 : les rues pavoisées de drapeaux, les bâtiments réquisitionnés pour accueillir les soldats blessés, mais aussi les théâtres, dont les programmes sont plus ou moins dictés par l'actualité, la guerre s'immisce dans tous les domaines de la ville, même quand elle se déroule au loin. Les problèmes d'approvisionnement et de sous-alimentation touchent de plein fouet les populations civiles de Prague, de Vienne et de Mannheim. La violence s'impose dans la vie quotidienne, comme le montre Ulrich Niess à propos de cette dernière : 6200 jeunes hommes de la ville sont morts au front, la moitié avait moins de 24 ans; 10000 habitants seraient morts des suites de la sous-alimentation et des épidémies. La violence se prolonge dans les années d'après-guerre, qui voient se multiplier émeutes et pillages, mais aussi les divorces et les violences domestiques. Les chiffres sont plus impressionnants encore pour Vienne, dont Alfred Poser livre un portrait ponctué de citations de Die letzten Tage der Menschheit de Karl Kraus. Les mêmes étapes se répètent : euphorie des premiers jours, qui laisse vite place à l'effroi devant l'arrivée des premiers milliers de blessés, les rumeurs, l'augmentation des prix, les listes des morts et des disparus ; 260000 blessés stationnent à Vienne en mars 1915, de même que 200 à 250000 réfugiés venus de Galicie et de Pologne.

La guerre modifie ainsi la physionomie des villes, mobilisées au service de l'effort de guerre national. Claire Morelon livre ainsi le passionnant tableau d'une Prague pavoisée de drapeaux et analyse l'enjeu des couleurs affichées, particulièrement important dans la troisième ville de l'Empire austro-hongrois, où se côtoient germanophones et Tchèques. Observant que la loyauté à l'Empire et les sentiments nationaux s'exprimaient simultanément dans l'espace public, C. Morelon conclut que les individus ont pleinement participé à la mobilisation patriotique, qui n’a donc pas relevé seulement d'ordres donnés du haut vers le bas par la bureaucratie impériale.

Dans un article particulièrement intéressant, Eva Krivanec montre quant à elle comment la guerre a en grande mesure décidé des programmes des théâtres européens. Si, dans les premiers jours, « le théâtre n'est plus dans les salles ordinaires, celles-ci ne [pouvant] pas donner des spectacles aussi édifiants que ceux qui s'offrent à chaque minute devant nos yeux », comme le relate un critique de théâtre parisien (p. 134), la réouverture des théâtres dès septembre et octobre 1914 est perçue comme un enjeu 
national; Gallieni, le gouverneur militaire de Paris, déclare ainsi : "Une ville sans spectacle est une ville vaincue » (p. 136). Les spectacles seront en revanche étroitement surveillés : la censure abolie en 1906 est réinstaurée, et les programmations ellesmêmes sont enrôlées dans l'effort de guerre, comme en témoigne le boycott général des dramaturges des pays ennemis. S'esquisse aussi dès 1917 une expérimentation formelle qui remet en cause les formes dramaturgiques traditionnelles ainsi que l'horizon d'attente des spectateurs: le ballet Parade est un "électrochoc", de même que le «drame surréaliste» d'Apollinaire monté en juin 1917, Les Mamelles de Tirésias. L'auteure s'attarde enfin sur le cirque Sarrasani qui proposait des spectacles aux titres explicites : Europa in Flammen et Torpedo - los! sont d'énormes productions, mettant en scène des moyens techniques dernier cri pour représenter des événements politiques et militaires : l'invasion de la Belgique, la guerre des sous-marins, le bombardement de Londres montent ainsi sur scène, dans de vastes ensembles chorégraphiques proposant un spectacle sensationnel. Les scènes de théâtre européennes subissent ainsi l'influence directe du conflit : elles sont enrôlées dans l'effort de guerre au nom de la propagande tout en donnant corps à cette "danse sur un volcan » qu'aura été l'expérience du conflit mondial.

Les deux dernières contributions s'intéressent à la mémoire urbaine de la Première Guerre mondiale. Élise Julien compare son inscription dans l'espace parisien et berlinois. Si les deux capitales ont été très fortement marquées par la guerre, elles ont des expériences très différentes du conflit. Paris est perçu comme une ville du front, lieu de la mobilisation industrielle et patriotique - et recevra la Croix de Guerre, au même titre que les villes détruites du nord et de l'est. Érigée au rang de symbole de la défense nationale, la capitale est plus que jamais considérée comme le cœur de la nation. À Berlin, le souvenir de la guerre est très lié à celui du blocus et des difficultés d'approvisionnement - ce qui rend sa représentation bien plus difficile. L'auteure montre bien comment la mémoire de la guerre dépend de la façon dont cette dernière a été vécue, tout en soulignant combien les associations locales ont été plus efficaces dans l'inscription spatiale d'une mémoire urbaine que les autorités municipales ellesmêmes.

Alexandra Kaiser s'intéresse pour finir à la mémoire des victimes civiles de la guerre à Karlsruhe, la ville qui a compté le plus de victimes d'attaques aériennes (168), dont la plupart sont mortes le 15 juin 1915 (30 morts) et le 22 juin 1916 (120, dont 71 enfants, la bombe étant tombée tout près d'un cirque). Expérience nouvelle à laquelle il s'agit de donner un sens et une forme: comment commémorer les victimes civiles aléatoires d'une attaque venue du ciel ? Faut-il les considérer comme des victimes de la guerre ou comme des victimes pour la patrie ? Cette oscillation interroge rien moins que le sens donné à la mort en temps de guerre. En étudiant précisément leur enterrement, A. Kaiser montre la portée de ces interrogations. La tombe collective de ces victimes n'est pas intégrée au cimetière monumental des soldats, mais elle en est toute proche, constituant une entité singulière. La mémoire de ces victimes est pourtant peu à peu éclipsée par celle des soldats tombés au front: ces morts par hasard n'entraient pas dans le modèle interprétatif du sacrifice pour la patrie.

Comme dans tous les volumes collectifs, les contributions sont inégales, certaines se contentant de retracer des étapes bien connues de la vie "de l'arrière ». L'éclairage local se révèle pourtant particulièrement efficace pour incarner ce pan de l'histoire 
urbaine et l'inscription de la guerre, au présent et au passé, dans le tissu de la ville et dans la vie quotidienne des citadins.

INDEX

Index chronologique : Époque contemporaine

Thèmes : Histoire des villes et des régions

\section{AUTEURS}

\section{AURORE PEYROLES}

Post-doctorante de l'IFRA (Frankfurt am Main) 